\title{
Efecto de Antígenos de Clostridium perfringens y Ácido Retinoico sobre la Expresión de IgA en la M ucosa Intestinal de Crías de Alpacas (Vicugna pacos)
}

\author{
EfFect of Clostridium perfringens Antigens ANd ACId Retinoic on IgA Expression \\ in the Intestinal Mucosa of Newborn Alpacas (Vicugna pacos)
}

\author{
Roberto Lázaro B. ${ }^{1}$, Alberto Manchego S. ${ }^{1,4}$, Danilo Pezo C. ${ }^{3}$, Juan More B. ${ }^{1}$, \\ Gina Castro S. ${ }^{1}$, Juan Siuce M. ${ }^{1}$, Nieves Sandoval C. ${ }^{2}$
}

\section{Resumen}

\begin{abstract}
El objetivo del estudio fue comparar los niveles de expresión relativa del gen del exón 1 de la IgA en el epitelio intestinal de las crías de alpacas tratadas y no tratadas con antígenos de Clostridium perfringens más ácido holotransretinoico (ATRA) por vía oral. Se muestrearon 32 animales: 14 tratados ( 6 de 1 día de edad y 8 entre 7 y 14 días de edad) y 18 no tratados ( 10 de 1 día de edad y 8 entre 7 y 14 días de edad). Los animales fueron sacrificados a la semana del tratamiento, se recolectó un segmento de $2 \mathrm{~cm}$ de yeyuno y se almacenó a $-196{ }^{\circ} \mathrm{C}$. De cada muestra se realizó la extracción de ARN total con el método de TRIzol® y luego se realizó la técnica del RT PCR a tiempo real empleando oligonucleótidos diseñados para la detección del exón 1 del gen de inmunoglobulina A (IgA) de alpaca. La cuantificación de la expresión relativa mediante normalización con ARNm del gen GAPDH y los cálculos de la expresión relativa se realizaron utilizando el

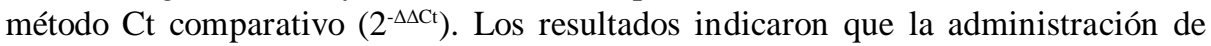
antígenos de $C$. perfringens y ATRA ejerce un efecto inmunomodulador positivo sobre la expresión de IgA en mucosa intestinal de crías de alpacas tratadas. Los animales tratados de ambos grupos etarios presentaron mayor producción de ARNm de IgA en relación a los animales controles $(\mathrm{p}<0.05)$.
\end{abstract}

Palabras clave: camélidos sudamericanos, Clostridium perfringens, inmunoglobulina A, ácido retinoico, RT-PCR Tiempo Real, cuantificación relativa

\footnotetext{
${ }^{1}$ Laboratorio de Microbiología y Parasitología Veterinaria, ${ }^{2}$ Laboratorio de Histología, Embriología y Patología Veterinaria, Facultad de Medicina Veterinaria, Universidad Nacional Mayor de San Marcos, Lima, Perú

${ }^{3}$ Estación Experimental del Centro de Investigación IVITA, Maranganí, Cusco, Perú

${ }^{4}$ E-mail: amanchegos@gmail.com
}

Recibido: 4 de setiembre de 2014

Aceptado para publicación: 30 de abril de 2015 
The aim of this study was to compare the relative expression levels of the gene from exon 1 of the IgA in the intestinal epithelium of baby alpacas untreated and treated orally with Clostridium perfringens antigens and all-trans retinoic acid (ATRA). Thirty two animals were sampled: 14 treated (6 of 1 day old and 8 between 7-14 days old) and 18 untreated ( 10 of 1 day old and 8 between 7-14 days old). The animals were slaughtered after one week of the treatment and $2 \mathrm{~cm}$ of the jejunum was collected and kept at $-196{ }^{\circ} \mathrm{C}$. Total RNA was extracted from each sample using the TRIzol@ method and complementary DNA (cDNA) was synthesized. PCR and RT-PCR Real Time were conducted using specific oligonucleotides designed for the detection of exon 1 of the region $\mathrm{Fc} \operatorname{IgA}$ in the alpaca. The quantification of the relative expression by normalization with GAPDH gene mRNA and the relative expression calculations were performed using the comparative $\mathrm{Ct}$ method $\left(2^{\Delta \Delta \mathrm{Ct}}\right)$. The results showed that the administration of $C$. perfringens antigens and ATRA exerts positive immunomodulatory effect of IgA on the intestinal mucosa of treated baby alpacas. Animals of both age groups showed higher IgA mRNA production with respect the non-treated animals $(\mathrm{p}<0.05)$.

Key words: South American camelids, Clostridium perfringens, immunoglobulin A, retinoic acid, Real Time RT-PCR, relative quantification

\section{INTRODUCCIÓN}

El Perú es el principal productor de camélidos sudamericanos (CSA) del mundo con más de cinco millones de cabezas, teniéndose 3.6 millones de alpacas que representan el $85 \%$ de la población mundial (MINAG, 2006). Las características naturales propias de los CSA favorecen una crianza productiva y rentable en las zonas alto andinas del Perú (Brenes et al., 2001). Sin embargo, la mortalidad de las crías de alpacas debido a enfermedades infecciosas causantes de neumonías y diarreas son los principales problemas sanitarios.

La enterotoxemia es una de las principales enfermedades infecciosas que afectan a los CSA, causando mortalidades de hasta $70 \%$ en crías de alpacas de 2 a 3 semanas de edad (Ramírez, 1991). La enterotoxemia es causada por las toxinas del Clostridium perfringens tipo A y C (Moro, 1987). Ell tipo A el de mayor importancia epidemiológica (Prehn et al., 1999), en tanto que los casos de tipo D, principal patógeno de bovinos y ovinos, solo se han registrado en camélidos criados en EEUU (Fowler, 1998). Las principales toxinas presentes en la enterotexemias de las crías de alpacas son la toxina alfa (CpPLC), enterotoxina (CPE) y $\beta 2$. La muerte súbita del animal transcurre luego de un breve periodo clínico, donde ocurre una toxemia focalizada a nivel del intestino delgado, pasando a una toxemia generalizada que causa lesiones del endotelio vascular y sistema nervioso (Novoa y Flórez, 1991).

No existe un producto farmacológico que contrarreste el efecto de las toxinas generadas por la bacteria; sin embargo, se emplean antibióticos como un control paliativo contra las diarreas secundarias para reducir la diseminación de bacterias oportunistas (Ortiz, 1988). Asimismo, el cambio de los animales a otros dormideros y canchas (pastizales) puede ayudar a paliar la enfermedad una vez establecida la epizootia (Moro, 1987).

Hay experiencias preliminares en el uso de vacunas anticlostridiales para reducir la mortalidad neonatal en alpacas (Moro, 1987). Los mejores resultados han sido con el uso de vacunas (anacultivo) con cepas de $C$. 
perfringens tipos A, B, C y D, predominantemente de origen ovino, donde se pudo reducir la mortalidad neonatal asociada a enterotoxemia desde $19.5 \%$ en el año 2000 a 7.2, 9.1 y $1.0 \%$ para los años 2001, 2002 y 2003, respectivamente (Yaya y Rosadio, 2005).

El ácido retinoico es un compuesto derivado de la vitamina $\mathrm{A}$, que es producido a nivel de la mucosa intestinal de los animales (Blomhoff R y Blomhoff $\mathrm{H}, 2006$ ) y es necesario para estimular a los linfocitos B presentes en los tejidos linfoides asociados a mucosas. Junto con el factor de crecimiento transformante $\beta$ (TGF $\beta$ ), induce la proliferación de linfocitos $\mathrm{B}$ para producir inmunoglobulina A ( $\operatorname{Ig} \mathrm{A})$ en estos tejidos (Maruya et al., 2011).

En el presente estudio se evaluó un programa de vacunación anticlostridial utilizando componentes bacterianos y antigénicos de $C$. perfringens en conjunto con una isoforma del ácido retinoico en crías de alpacas de dos grupos etarios, cuantificando la expresión de la IgA a través del método CT comparativo $\left(2^{-\Delta \Delta \mathrm{Ct}}\right)$.

\section{MATERIALES Y MéTodos}

\section{Preparación de la Vacuna}

\section{Aislado de antígenos proteicos}

Los componentes antigénicos clostridiales fueron obtenidos a partir del cultivo de muestras intestinales tomadas de alpacas que murieron con cuadros clínicos y lesiones compatibles con enterotoxemia. Se utilizó caldo tioglicolato a $37^{\circ} \mathrm{C}$ por $72 \mathrm{~h} \mathrm{y}$, luego, se sembró en agar sangre de cordero al $5 \%$, incubado a $37{ }^{\circ} \mathrm{C}$ por $48 \mathrm{~h}$ en anaerobiosis (Anaerocult Gaspack). Los aislados se identificaron mediante pruebas bioquímicas. Para la precipitación de los antígenos clostridiales se empleó ácido tricloroacético (TCA) al 10\% en concentración final (Brown et al., 1989). La medición de la concentración de antígenos proteicos se realizó mediante el fluorómetro Qubit ${ }^{\circledR}$ (Life Technologies, EEUU), siguiendo las especificaciones del fabricante.

\section{Cálculo de las dosis}

La dosis de antígeno proteico $(12.89 \mathrm{mg})$ se calculó de acuerdo a concentraciones de vacunas comerciales, determinadas mediante el fluorómetro Qubit TM.

La dosis de ácido holotransretinoico (ATRA) y dimetilsulfóxido (DMSO) fue hallada a partir de una proporción correspondiente a $5 \mathrm{mg}$ ATRA por cada $1.664 \mathrm{ml}$ de DMSO. En esta relación, el ATRA se satura en DMSO y permite su dilución, el mismo que equivale a $10 \mathrm{mM}$ de ATRA (Yu et al., 2005). La dosis usada correspondió a $0.1 \mathrm{mM}$ de ATRA por cada kilogramo de peso vivo en un volumen final de $5 \mathrm{ml}$. La vacuna fabricada se conservó en refrigeración hasta su uso.

\section{Protocolo Experimental}

Se utilizaron crías de alpacas de un rebaño criado sobre pastos naturales en Maranganí, Cusco, Perú, sin tener en consideración el sexo o peso. Los animales fueron organizados en dos grupos (A y B) y recibieron la vacuna por vía oral (VO), según se muestra en el Cuadro 1. La vacunación se realizó en el mismo lugar de crianza durante la temporada de lluvias. El número de animales utilizados en el experimento se determinó con base a la disponibilidad de crías en el rebaño. El tratamiento se impartió en dos tiempos: la primera dosis se hizo de acuerdo al grupo etario correspondiente y la segunda dosis a la semana posterior. Los animales fueron sacrificados una semana posterior a la segunda dosis (Grupo A a los 14 días y Grupo B entre 21 y 28 días de edad). Los animales de los grupos control fueron sacrificados a las mismas edades que los tratados. 
Cuadro 1. Distribución de los animales según programa de vacunación anticlostridial

\begin{tabular}{cclc}
\hline Grupos & $\begin{array}{c}\text { Grupo etario } \\
\text { (en días) }\end{array}$ & Condición & $\begin{array}{c}\text { Animales } \\
\left(\mathrm{N} .^{\circ}\right)\end{array}$ \\
\hline \multirow{2}{*}{$\mathrm{A}$} & 1 & Tratados $^{1}$ & 6 \\
& & No Tratados & 10 \\
\multirow{2}{*}{$\mathrm{B}$} & \multirow{2}{*}{$7-14$} & Tratados $^{1}$ & 8 \\
& & No Tratados & 8 \\
\hline Total & & & 32 \\
\hline
\end{tabular}

${ }^{1}$ Antígenos de Clostridium perfringens más ácido holotransretinoico

El manejo y posterior sacrificio de los animales estuvieron sujetos a los principios directrices internacionales para la investigación biomédica que implique el uso de animales (CIOMS, 2012). Para el sacrificio de los animales se utilizó un protocolo basado en 1.5 $\mathrm{mg} / \mathrm{kg}$ de xilacina (Rompun®) y $7.5 \mathrm{mg} / \mathrm{kg}$ de ketamina (Vetalarß) i.m. (Urquieta et al., 1992), seguido por una sobredosis de $50 \mathrm{mg} /$ kg e.v. de pentobarbital sódico (Halatal $\left.{ }^{\circledR}\right)$.

Se tomaron muestras de yeyuno $(2 \mathrm{~cm})$, se depositaron en crioviales y se almacenaron en nitrógeno líquido $\left(-196^{\circ} \mathrm{C}\right)$.

\section{Cuantificación Relativa de IgA}

\section{Extracción de RNA mensajeros totales}

Se empleó TRIzol® LS Reagent (Invitrogen) de acuerdo a las especificaciones del fabricante. El producto final fue reconstituido en $70 \mu \mathrm{l}$ de agua ultrapura. Las muestras fueron conservadas a $-70{ }^{\circ} \mathrm{C}$ para su posterior uso en la síntesis de ADN complementario (cDNA).

Sintesis de ADN complementario (cDNA)

Se utilizó el kit DyNAmoTM SYBR Green 2-step qRT-PCR (Thermo Fisher Scientific, EEUU), siguiendo las especificaciones del fabricante. Se adicionaron alícuotas de $2 \mu 1$ de ARN total extraído por muestra a $18 \mu \mathrm{l}$ del master mix preparado previamente. El protocolo del Termociclador Thermal Cycler PTC-200 Chromo 4 (MJ Research, EEUU) para la síntesis de cDNA fue: $25^{\circ} \mathrm{C}$ por $10 \mathrm{~min}, 37^{\circ} \mathrm{C}$ por $30 \mathrm{~min}, 85^{\circ} \mathrm{C}$ por 5 min y, finalmente, $4^{\circ} \mathrm{C}$ hasta enfriar la muestra. El producto fue conservado a $-70{ }^{\circ} \mathrm{C}$ para su posterior uso en el RT PCR Tiempo Real.

Selección de oligonucleótidos de IgA y GAPDH

La amplificación de un segmento de ARN mensajero del Exón 1 de IgA se realizó empleando los oligonucleótidos diseñados por el programa Primer3 Output (www.primer3.com), a partir de la secuencia publicada en el Banco de Genes (GenBank), para la línea germinal de alpaca (AM773729.1). Del mismo modo, se utilizó el programa BLAST del NCBI (www.ncbi.nlm.nih.gov/blast/) para encontrar las regiones conservadas de la línea germinal de alpaca realizando comparaciones y demostrando su especificidad (Dionisio, 2012).

Se utilizaron oligonucleótidos específicos para el gen GAPDH (gliceraldehído 3 fosfato deshidrogenasa) (Patil et al., 2004) usado como control endógeno (Cuadro 2). Realizado el análisis BLAST, los oligonucleótidos diseñados permitieron identificar parte de un producto de 309 nucleótidos 
Cuadro 2. Secuencia de oligonucleótidos del Exón 1 de la $\operatorname{IgA}$ y GAPDH (gliceraldehído 3-fosfato deshidrogenasa)

\begin{tabular}{cclc}
\hline Gen & $\begin{array}{c}\text { Longitud } \\
(\mathrm{pb})\end{array}$ & \multicolumn{1}{c}{ Secuencia } & $\begin{array}{c}\mathrm{T}^{\mathrm{o}} \\
\text { hibridación }\end{array}$ \\
\hline Ig A & 164 & $\begin{array}{l}\text { F: 5'AACGTGTCCGTCATGGACTT 3' } \\
\text { R: 5' GGTAGTTGGGCATGTTGATC 3' }\end{array}$ & $60.4^{\circ} \mathrm{C}$ \\
GAPDH & 356 & $\begin{array}{l}\text { F: 5' GTGAAGGTCGGAGTGAACG 3' } \\
\text { R: 5' GAGATGATGACCCTCTTGGC 3' }\end{array}$ & $60.0^{\circ} \mathrm{C}$ \\
\hline
\end{tabular}

del locus IgH en la región del gen IgHv de la secuencia de la línea germinal de alpaca que comparte una identidad de $88 \%$ con el ARNm de la IgA del cerdo (Sus scrofa).

\section{PCR Tiempo Real}

Mediante la técnica RT-PCR Tiempo Real se identificó al gen GAPDH (housekeeping gene), el cual sirvió de control interno y para realizar la cuantificación relativa por el método Thermo Fisher Scientific (EEUU), siguiendo las especificaciones del fabricante. Se tomaron $18 \mu \mathrm{l}$ del master mix preparado previamente y se agregó $2 \mu 1 \mathrm{del}$ producto de la síntesis de cDNA. El protocolo para el termociclador consistio en: $95{ }^{\circ} \mathrm{C}$ por $15 \mathrm{~min}, 94^{\circ} \mathrm{C}$ por $10 \mathrm{~min}, 55^{\circ} \mathrm{C}$ por 30 min, $72{ }^{\circ} \mathrm{C}$ por $30 \mathrm{~min}$, y repetición por 40 veces a partir del segundo paso. Los resultados de la amplificación fueron determinados según la curva de amplificación (Cts) de los productos y la temperatura de disociación (Tm) de los mismos. El software empleado fue el Opticon Monitor 2 v. 2.03.

\section{Resultados}

La prueba de RT PCR tiempo real determinó la presencia de los RNA mensajeros del exón 1 de la inmunoglobulina A en todos los animales (controles y vacunados). Los productos obtenidos tuvieron una temperatura de disociación alrededor de $85.7^{\circ} \mathrm{C}$ (Fig. 1). Asimismo, se determinó que los animales vacunados presentan una mayor expresión del gen de la inmunoglobulina A en la mucosa del yeyuno que los controles no vacunados. El análisis de cuantificación relativa evidenció una mediana de $9020.6 \pm 21.2$ unidades (teniendo la expresión del exón 1 de la IgA del calibrador, animal recién nacido, como la unidad) para los animales tratados y de 55.3 \pm 12.8 unidades para los no tratados. En el análisis estadístico se encontró diferencia significativa entre grupos ( $\mathrm{p}=0.0120$ ) (Fig. 2).

En el análisis de cuantificación relativa dentro de grupos etarios, se obtuvo una mediana de 9182.8 unidades para los animales tratados y de 1193.38 unidades para los no tratados de un día de edad, con rangos de 11.5 y 6.7 para tratados y no tratados, respectivamente, mostrando una diferencia significativa $(\mathrm{p}=0.0499)$. En forma similar, se obtuvo una mediana de 4533.3 unidades para los animales tratados y de 6.3 unidades para los no tratados entre 7 y 14 días de edad $(\mathrm{p}=0.0115)$, con rangos de 11.5 y 5.5 para tratados y no tratados, respectivamente (Fig. $3)$. Este resultado determina que los animales vacunados desde el primer día de edad recibieron un estímulo suficiente para inducir una alta producción de inmunoglobulina A en la mucosa yeyunal de las crías de las alpacas. 


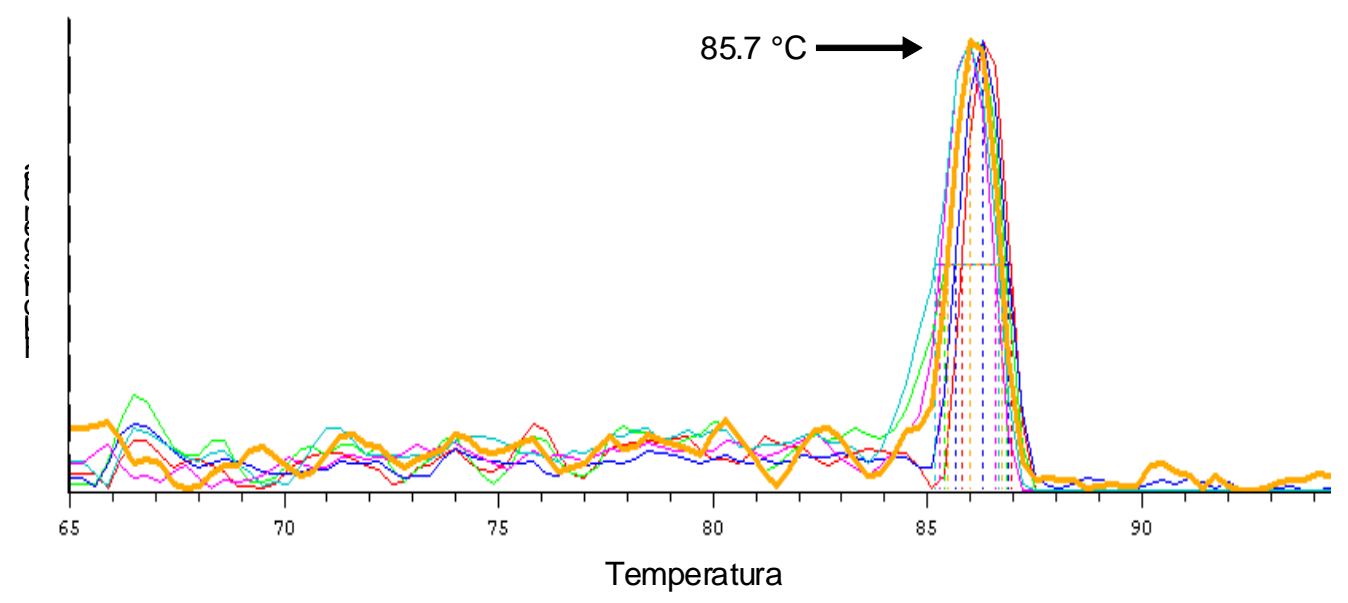

Figura 1. Análisis de temperatura de disociación (Tm) mediante RT-PCR Tiempo Real. Se evidencia la superposición de las curvas con un $\mathrm{Tm}$ de $85.7{ }^{\circ} \mathrm{C}$

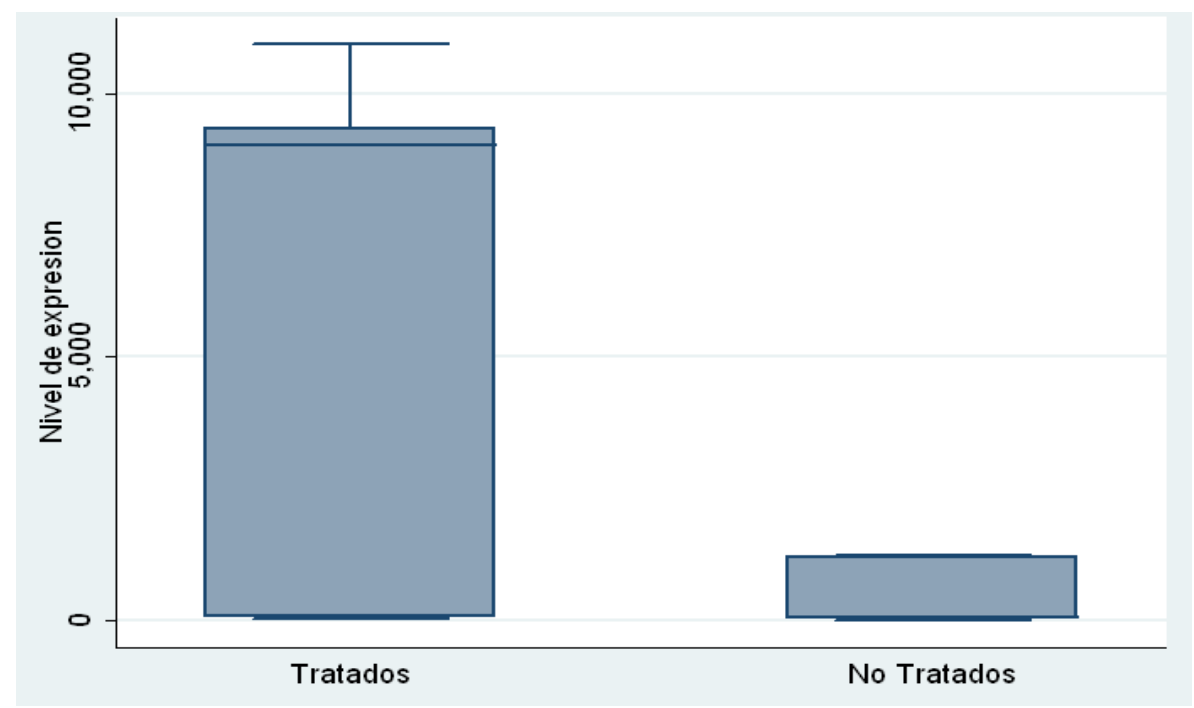

Figura 2. Expresión relativa de ARNm de Ig A en mucosa intestinal de crías de alpacas tratadas (antígenos de Clostridium perfringens más ATRA) y no tratadas

\section{Discusión}

El estudio permitió determinar el aumento de expresión relativa del exón 1 de la $\operatorname{IgA}$ en mucosa intestinal de crías de alpacas tratadas con respecto a sus controles. La Tm de los productos de la RT PCR tiempo real fue similar a la encontrada en un estudio de cinética de expresión de la $\operatorname{IgA}$ en crías de alpacas clínicamente sanas (Dionisio, 2012).
Se comprueba el rol que desempeñaría ATRA como inmunomodulador positivo hallado en otros estudios con animales de laboratorio (Kang et al., 2007; Ma y Ross, 2009). Los resultados muestran una mayor expresión del exón 1 de la IgA en animales tratados con respecto a los controles. Estos resultados estarían correlacionados con estudios en ratones con dietas basales en vitamina A, presentando alteraciones de señalización de TGF- $\beta 1$ por descenso en su expresión, así 


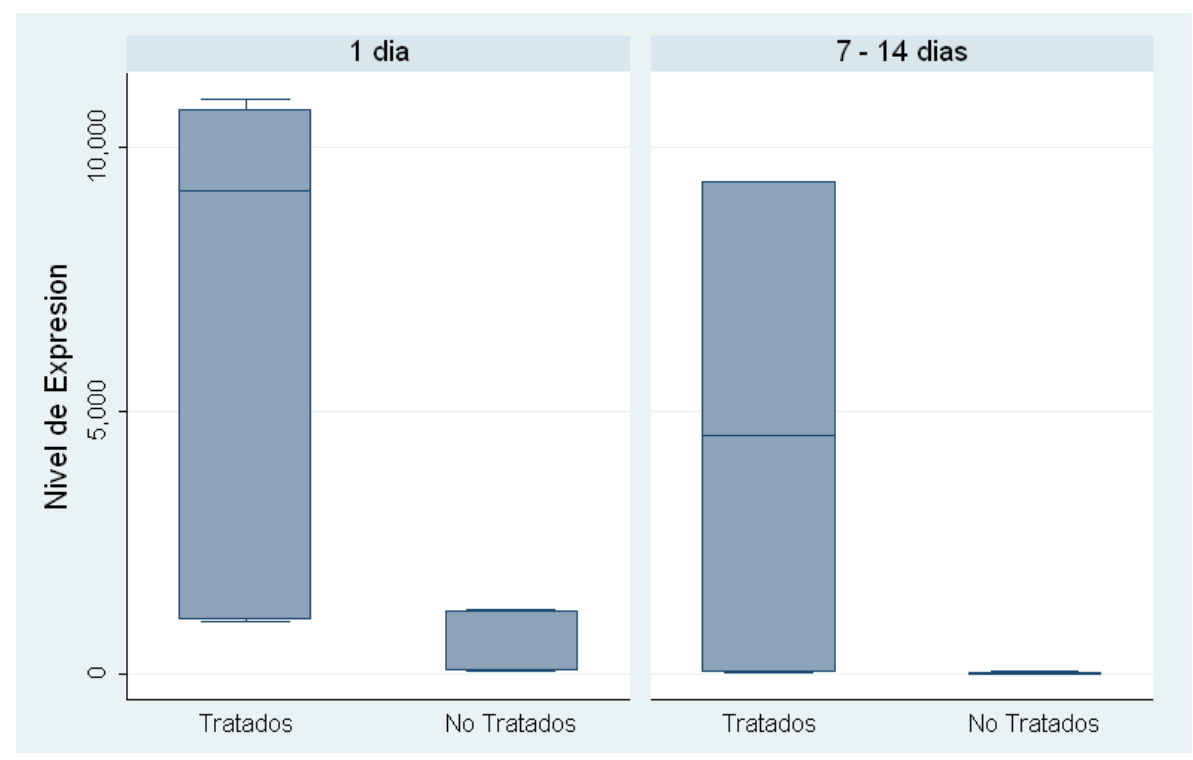

Figura 3. Expresión relativa de ARNm de Ig A en mucosa intestinal de crías de alpacas tratadas (antígenos de Clostridium perfringens más ATRA) y no tratadas, según grupo etario

como de su receptor celular, lo que alteraría el cambio de clase hacia $\operatorname{IgA}(\mathrm{C} \alpha / \mathrm{I} \alpha)$ (Borsutzky et al., 2004; Cerutti, 2008).

El uso del ATRA en la preparación del inmunógeno en estudio permitió estimular la expresión del gen IgA en la mucosa intestinal. Se ha demostrado en ratones que el ácido retinoico (obtenido del metabolismo de la vitamina A a nivel de la mucosa intestinal) estimula la expresión de los receptores homing ( $\alpha 4 \beta 7$ y ccr9) en las células $T$, los que se unen a sus ligandos (MadCAM-1 y CCL25) expresados por las vénulas poscapilares del intestino, esenciales para direccionar el tráfico linfocitario hacia la mucosa intestinal. Esto permitiría un mayor número de linfocitos $\mathrm{T}$ capaces de estimular a los linfocitos B, incrementando la producción y secreción de IgA hacia el lumen intestinal. Además, facilitaría una retroalimentación positiva para la presentación antigénica por células presentadoras de antígenos a una población de linfocitos B renovada, beneficiando a su vez la expresión contra dichos antígenos en otras mucosas (Ross, 1992; Elias et al., 2008).
La mayor expresión del exón 1 de la IgA en los animales tratados de 1 día de edad en relación con los controles sería por la presencia de un lumen intestinal menos expuesto a factores y eventos que afectarían la eficiente presentación y absorción de la vacuna, al tener mayor superficie de contacto y mejor captación antigénica por la células $\mathrm{M}$ (Fig. 3). Por otro lado, la mayor expresión encontrada en el grupo etario B podría deberse al mejor desarrollo de los componentes del GALT para su mejor captación y presentación antigénica.

El desarrollo de una mejor respuesta Th2, característico en condiciones de presentación antigénica bacteriana y parasitaria, donde el perfil de citoquinas se enmarca con mayor producción de IL-4 e IL-10, e inhibiendo las citoquinas INF $\gamma$ y FNT $\alpha$, desenlaza en una respuesta inmunitaria humoral a nivel de mucosas representada con la mayor producción de IgA. Sin embargo, estudios previos reportan el perfil Th1 como dominante en mucosa intestinal de crías de alpacas, lo que estaría supeditado a un traba- 
jo retrospectivo, donde las condiciones, tiempo y tipo de infección serían ajenas al control del investigador (Chiok, 2012).

Por otro lado, para mantener un ambiente luminal óptimo y preparado para enfrentar a noxas, es imprescindible que las respuestas tipo Th1 y Th2 se orquesten para ejecutar la mejor respuesta inmunitaria acorde al tipo antigénico (Yu et al., 2005). En tal sentido, el DMSO ha sido probado en ratones donde jugaría un papel adyuvante administrado junto con antígenos proteicos, surcando una respuesta Th1, evidenciada por la producción de IL6 Y FNT- $\alpha$ (Xing y Remick, 2005).

La metodología experimental diseñada para el presente estudio enmarca los resultados en un protocolo donde la administración de ATRA y antígenos de $C$. perfringens se hizo en forma conjunta, no permitiendo evaluar el efecto por separado de cada componente. Sin embargo, en otros estudios se ha comprobado la capacidad de ATRA para modular eventos que desarrollarían la mayor expresión de $\operatorname{IgA}$ en mucosa (Chen et al., 2011). Por otro lado, el uso de antígenos de $C$. perfringens totales, que incluyen antígenos somáticos y exotoxinas, canalizaría mejor la producción de distintos tipos de IgA específicas para los distintos antígenos clostridiales para una eficiente protección. Preparaciones combinadas de antígeno somático bacteriano y el antígeno de la toxina de Vibrio cholerae se han reportado para actuar de forma sinérgica en la estimulación de la inmunidad en animales de laboratorio, determinando que el efecto protector combinado está más cerca del producto que de la suma de los efectos individuales de protección (Svennerholm y Holmgren, 1976).

\section{Conclusiones}

La administración conjunta de ATRA $(0.067 \mathrm{UI} / \mathrm{kg})$ y antígenos de Clostridium perfringens $(12.89 \mathrm{mg}$ ) eleva los niveles de Ig A en mucosa intestinal de crías de alpacas.

\section{Agradecimientos}

Los autores agradecen a los criadores alpaqueros de las comunidades de la provincia de Canchis, Cusco, por su colaboración para la adquisición de los animales utilizados. El trabajo fue financiado por el Fondo Nacional de Innovación en Ciencia y Tecnología (FINCYT - PIBAP).

\section{Literatura Citada}

1. Blomhoff R, Blomhoff HK. 2006. Overview of retinoid metabolism and function. J Neurobiol 66: 606-630. doi: 10.1002/neu.20242

2. Borsutzky S, Cazac B, Roes J, Guzman C. 2004. TGF- $\beta$ receptor signaling is critical for mucosal $\operatorname{IgA}$ responses. J Immunol 173: 3305-3309.

3. Brenes E, Madrigal K, Pérez, F, Valladares K. 2001. El cluster de los camélidos sudamericanos en Perú: diagnóstico competitivo y recomendaciones estratégicas. [Internet]. Disponible en: http://infoalpacas.com.pe/el-cluster-decamelidos-en-peru-diagnostico-competitivo-y-recomendaciones-estrategicas/

4. Brown RE, Jarvis KL, Hyland KJ. 1989. Protein measurement using bicinchoninic acid: elimination of interfering substances. Anal Biochem 180: 136-139.

5. Cerutti A. 2008. The regulation of IgA class switching. Nat Rev Immunol 8: 421-434.

6. Chen Q, Mosovsky K, Ross C. 2011. Retinoic acid $\alpha$-galactosylceramide differentially regulate $B$ cell activation in vitro and augment antibody production in vivo. Clin Vaccine Immunol 18: 10151020.

7. Chiok K. 2012. Efecto de citoquinas de la respuesta Th1(INF y e IL2) y Th2(IL4 e IL10) en mucosa intestinal de crías de alpacas (Vicugna pacos) sanas y con enteropatías. Tesis de Magíster. Lima: Univ Nac Mayor de San Marcos. 120 p. 
8. [CIOMS] Consejo de Organizaciones Internacionales de las Ciencias Médicas. 2012. International guiding principles for biomedical research involving animals. http://www.cioms.ch/ images/stories/CIOMS/IGP2012.pdf

9. Dionisio J. 2012. Cinética de expresión de inmunoglobulina Aen el epitelio intestinal de crías de alpaca (Vicugna pacos). Tesis de Médico Veterinario. Lima: Univ Nac Mayor de San Marcos. 59 p.

10. Elias K, Laurence A, Davidson T, Stephens G, Kanno Y, Shevach E, O'Shea J. 2008. Retinoic acid inhibits Th17 polarization and enhances FoxP3 expression through a Stat-3/Stat-5 independent signaling pathway. Blood 111: 1013-1020.

11. Fowler M. 1998. Medicine and surgery of South American camelids. $2^{\text {nd }}$ ed. Iowa, USA: Iowa State University Press. 391 p.

12. INEI [Instituto Nacional de Estadística e Informática]. 2012. IV Censo Nacional Agropecuario 2012. [Internet]. Disponible en: http://censos.inei.gob.pe/ cenagro/tabulados/

13. Ma Y, Ross A. 2009. The anti-tetanus immune response of neonatal mice is augmented by retinoic acid combined with polyriboinosinic:polyribocytidylic acid. Proc Natl Acad Sci USA 102: 13556-13561.

14. Maruya M, Suzuki K, Fujimoto H, Miyajima $M$, Kanagawa $O$, Wakayama T, Fagarasan S. 2011. Vitamin A-dependent transcriptional activation of the nuclear factor of activated T cells $\mathrm{c} 1$ (NFATc1) is critical for the development and survival of B1 cells. Proc Natl Acad Sci USA 108: 722727. doi: $10.1073 /$ pnas. 1014697108

15. Moro M. 1987. Enfermedades infecciosas de las alpacas. Diarrea bacilar o enterotoxemia de las crías de las alpacas. Rev Camélidos Sudam 4: 8-13.

16. Novoa C, Flórez A. 1991. Producción de rumiantes menores: alpacas. Lima: Ed Rerumen. $331 \mathrm{p}$.
17. Ortiz S. 1988. Evaluación de algunos métodos de control de la mortalidad en crías de alpaca (Lama pacos) en explotaciones familiares. Tesis de Médico Veterinario. Lima: Univ Nac Mayor de San Marcos. 58 p.

18. Patil A, Hughes A, Zhang G 2004. Rapid evolution and diversification of mammalian $\alpha$-defensins as revealed by comparative analysis of rodent and primate genes. Physiol Genomics 20: 1-11.

19. Prehn N, Saez M, Arraigada M. 1999. Estudios microbiológicos y clínicos de enterotoxemia por Clostridium perfringens en camélidos sudamericanos. Res II Congreso Mundial sobre Camélidos. Lima, Perú.

20. Ramírez A. 1991. Enfermedades infecciosas. En: Fernández-Baca $\mathrm{S}$ (ed). Avances y perspectivas del conocimiento de los camélidos sudamericanos. Santiago de Chile: FAO. p 263-324.

21. Ross A. 1992. Vitamin A: relationship to immunity and the antibody response. Proc Soc Exp Biol Med 200: 303-320.

22. Svennerholm AM, Holmgren J. 1976. Synergistic protective effect in rabbits of immunization with Vibrio cholerae lipopolysaccharide and toxin/toxoids. Infec Immun 13: 735-740.

23. Urquieta B, Schiappacasse M, Raggi L, Martínez R, Ferguson JG. 1992. Sedación, inmovilización y anestesia com xilacina-ketamina en vicuña (Vicugna vicugna). Avances Cs Vet 7(2). [Internet]. Disponible en: http:// www.revistas.uchile.cl/index.php/ACV/ article/viewArticle/10425/10481

24. Xing L, Remick D. 2005. Mechanisms of dimethyl sulfoxide augmentation of IL.1B production. J Immunol 174: 61956202.

25. Yaya K, Rosadio R. 2005. Ensayo de tres programas de vacunación anticlostridial en alpacas. Rev Inv Vet Perú 16: 49-55.

26. Yu S, Xia M, Xu W, Chu Y, Wang Y, Xiong, S. 2005. All-trans acid biases immune response induced by DNA vaccine in a Th2 direction. Vaccine 23:5160-5167. 